\title{
СОВРЕМЕННЫЙ ПОДХОД К ХИРУРГИЧЕСКОМУ ЛЕЧЕНИЮ У ПАЦИЕНТОВ С ОСТРЫМ КОРОНАРНЫМ СИНДРОМОМ И С ПОРАЖЕНИЕМ СТВОЛА КОРОНАРНОЙ АРТЕРИИ
}

\section{MODERN APPROACH TO SURGICAL TREATMENT IN PATIENTS WITH ACUTE CORONARY SYNDROME WITH LESIONS OF THE LEFT CORONARY ARTERY}

A. Jalilov

Summary. Cardiovascular surgery is a fairly young and rapidly developing branch of medicine. In recent years, the latest surgical aids have been developed, huge steps have been taken in the anesthesiological support of operations, the range of resuscitation measures has expanded, allowing you to take care of extremely serious patients. In coronary surgery, the emphasis has shifted towards minimally invasive techniques that are most attractive to both patients and surgeons. In this article, we would like to reflect the evolution of treatment methods for patients with lesions of the left trunk of the coronary artery. A number of authors have found that surgical treatment not only reduces the clinical manifestations of acute coronal syndrome, but also significantly prolongs the life of patients [5]. However, the lack of randomization and evaluation of long-term results was a drawback of these studies. Therefore, the next step was to conduct long-term, multicenter, randomized trials that assessed life expectancy, quality and cost of treatment. However, in the arsenal of cardiologists, since 1977, a new treatment for coronary heart disease has appeared transluminal balloon angioplasty. This technique is actively developing and today most types of atherosclerotic lesions of the coronary bed are successfully corrected with the help of percutaneous angioplasty. Nevertheless, lesion of the left trunk of the coronary artery remains one of the few contraindications for angioplasty. This is due to the high mortality rate in acute occlusion of the LCA trunk, which occurs at the time of balloon inflation, while the risk in coronary artery bypass grafting does not exceed $5 \%$. This position is enshrined in the guidelines for percutaneous coronary angioplasty published by the American College of Cardiology / American Heart Association in 1993 [6]. But, despite the contraindication, transluminal balloon angioplasty is performed in a number of cases in patients with lesions of the LCA trunk. This category includes patients with the so-called "protected» left coronary artery trunk, which implies the presence of a functioning shunt into the left coronary artery system, patients with acute myocardial infarction, as well as those cases when acute occlusion of the trunk occurred at the time of diagnostic catheterization. Balloon angioplasty with stenting in such, often emergency cases, has made it possible to accumulate some experience in the interventional treatment of patients with lesions of the left coronary artery.

\author{
Жалилов Адхам Кахрамонович \\ К.м.н., Каршинский филиал республиканского \\ специализированного научно-практического \\ медицинского чентра кардиологии \\ Jalilov_adham@mail.ru
}

Аннотация. Сердечно-сосудистая хирургия является довольно молодой и стремительно развивающейся отраслью медицины. За последние годы разработаны новейшие хирургические пособия, сделаны огромные шаги в анестезиологическом обеспечении операций, расширился диапазон реанимационных мероприятий, позволяющих выхаживать крайне тяжелых больных. В коронарной хирургии акцент сместился в сторону малоинвазивных методик, наиболее привлекательных как для пациентов, так и для хирургов. В этой статье мне хотелось бы отобразить эволюцию методов лечения больных с поражением ствола левой коронарной артерии.

Тактика лечение этих пациентов, как и других больных с ишемической болезнью сердца, сводилась к медикаментозному и хирургическому. Вскоре был накоплен достаточный материал, позволивший провести первый ретроспективный анализ и сравнить результаты обоих методов лечения. Рядом авторов было установлено, что хирургическое лечение, не только уменьшает клинические проявления острого короанрного синдрома, но и существенно продлевает жизнь больным [5]. Однако, недостатком данных работ было отсутствие рандомизации и оценки отдаленных результатов. Поэтому следующим этапом было проведение длительных, многоцентровых, рандомизированных исследований, в которых оценивались продолжительность жизни, качество и стоимость лечения.

Так, в арсенале кардиологов, начиная с 1977 года, появилось новое средство лечения ишемической болезни сердца - транслюминальная баллонная ангиопластика. Эта методика активно развивается и на сегодняшний день большинство типов атеросклеротического поражения коронарного русла с успехом корректируется при помощи чрескожной ангиопластики. Тем не менее, поражение ствола левой коронарной артерии остается одним из немногих противопоказаний к проведению ангиопластики. Это связано с высокой летальностью при острой окклюзии ствола ЛКА, возникающей в момент раздувания баллона, тогда как риск при коронарном шунтировании не превышает 5\%. Данное положение закреплено в рекомендациях по чрескожной коронарной ангиопластике, опубликованных American College of Cardiology / American Heart Association в 1993 году [6]. Но, несмотря на противопоказание, транслюминальная баллонная ангиопластика выполняется в ряде случаев больным с поражением ствола ЛКА. К данной категории относятся пациенты с так называемым «защищенным» стволом ЛКА, что подразумевает под собой наличие функционирующего шунта в систему левой коронарной артерии, больные с острым инфарктом миокарда, а также те случаи, когда острая окклюзия ствола произошла в момент проведения диагностической катетеризации. 
Keywords: ischemic heart disease, acute coronary syndrome, trunk of the LCA.
Проведение баллонной ангиопластики со стентированием в подобных, нередко экстренных случаях, позволило накопить определенный опыт интервенционного лечения больных с поражением ствола ЛКА.

Ключевые слова: ишемическая болезнь сердца, острый коронарный синдром, ствол ЛКА.

ным» стволом ЛКА, что составило $0.4 \%$ от общего числа больных, которым была выполнена баллонная ангиопластика. Тяжелые сопутствующие заболевания послужили поводом для выбора чрескожной ангиопластики вместо коронарного шунтирования у 37 (51.1\%) пациентов. Тяжелое поражение периферических артерий было у 19 (26.8\%) пациентов, хронические обструктивные заболевания легких с резким снижением легочной функции - у 6 (8.5\%) больных, кардиомиопатия - у 4 (5.6\%), и злокачественные опухоли - у 1 (1.4\%). У 34 (47.9\%) человек выбор баллонной ангиопластики основывался в равной степени на предпочтении пациентов и благоприятной анатомии коронарных артерий, несмотря на отсутствие противопоказания к хирургическому лечению. В 23 случаях (32.4\%) была выполнена только баллонная ангиопластика, и в 46 (64.4\%) случаях использовались стенты. Через 1 год после процедуры выживаемость составила 98.6\% (погиб 1 больной). У 11 пациентов процедура осложнилась диссекцией ствола левой коронарной артерии, в одном случае была выполнена экстренная операция коронарного шунтирования. Ни у одного больного за время исследования не развился инфаркт миокарда. Одному пациенту (1.4\%) через 6 недель была выполнена повторная ангиопластика, тринадцати пациентам (18.3\%) в последующем было выполнено коронарное шунтирование $[9,11]$

В клинике Пастера в Тулузе с 1994 по 1999 год у 115 пациентов с «незащищенным» стволом ЛКА было выполнено стентирование. Непосредственный успех процедуры составил 100\%, госпитальная летальность была 3.5\%. Через 7 месяцев повторное стентирование потребовалось в 4\% случаев, а операция коронарного шунтирования выполнена в 2\% [12,13]. Авторами этой работы были сформулированы основные показания и противопоказания к стентированию «незащищенного» ствола ЛКА.

Показания к стентированию «незащищенного» ствола ЛКА:

1. Острая окклюзия ствола ЛКА.

2. Высокий риск или невозможность выполнения операции АКШ вследствие выраженного обструктивного поражения легких или «диализзависимой» почечной недостаточности.

3. Пациенты с поражением ствола левой коронарной артерии и многососудистым диффузным по- 
ражением, препятствующим адекватному шунтированию.

4. Пациенты с низким риском операции АКШ, с хорошей сократительной функцией левого желудочка, с локализацией поражения ствола в устье или в средней части.

Однако, по данным Marco J. с соавторами, даже опираясь на эти показания к стентированию, чрескожная ангиопластика выполняется лишь в 15\% случаев поражения ствола ЛКА, тогда как, подавляющему числу пациентов 71\% делают коронарное шунтирование, и оставшиеся 14\% лечатся медикаментозно. Таким образом, хирургическое вмешательство, по-прежнему остается основным методом лечения больных с поражением ствола левой коронарной артерии. $[13,14]$

Малоинвазивная реваскуляризация миокарда. В последние годы в коронарной хирургии акцент сместился в сторону снижения травматизации операций. Основными условиями выполнения стандартной операции коронарного шунтирования являются: срединная стернотомия, искусственное кровообращение, пережатие аорты и кардиоплегическая остановка сердца. Исключение одного или нескольких из этих факторов позволяет значительно снизить операционную травму и способствует более быстрому выздоровлению пациентов, а также позволяет уменьшить затраты на лечение. [15] Существует несколько основных видов миниинвазивной реваскуляризации миокарда. Техника Port-Access coronary surgery включает методику эндовазального бедренно-бедренного искусственного кровообращения с внутрипросветным пережатием аорты и введением кардиоплегического раствора. $[13,14]$

Операция выполняется на остановленном сухом сердце через специальные мини-порты полностью торакоскопически. Основным преимуществом данного метода является отказ от срединной стернотомии, однако проведение подобных операций возможно лишь у специально отобранной группы пациентов с однососудистым поражением сосудов передней стенки сердца. Другой вид операций миниинвазивной реваскуляризации миокарда Minimally Invasive Direct Coronary Artery Bypass (MIDCAB) выполняется через передне-боковую торокотомию в четвертом или пятом межреберье с резекцией реберного хряща, шунтирование выполняется на работающем сердце с использованием стабилизаторов миокарда. [16,19] Недостатками метода является: возможность шунтирования только 1-2 коронарных артерий, при необходимости перехода к ИК приходится выполнять экстренную стернотомию. Off - Pump Coronary Artery Bypass (OРCAB) наиболее распространенный вид миниинвазивной реваскуляризации миокарда. Операция выполняется через стан- дартную срединную стернотомию, что обеспечивает свободный доступ, как ко всем магистральным сосудам грудной клетки, так и ко всем коронарным артериям. Шунтирование выполняется без использования аппарата искусственного кровообращения и без кардиоплегии. Современные системы стабилизации и вертикализации миокарда позволяют обеспечить достаточную неподвижность участка миокарда в области анастомоза, а также дают возможность работать с любой коронарной артерией без существенного снижения гемодинамики. Единственным противопоказанием для операций ОРСАВ является необходимость выполнения коронарного шунтирования со вскрытием полости левого желудочка, что требуется при коррекции клапанного аппарата либо при наличии аневризмы левого желудочка. [3,4,8,14]

Стремление кардиохирургов отказаться от искусственного кровообращения связано с наличием большого числа побочных эффектов. К ним относятся: активация системного воспалительного ответа, коагулопатии, задержка жидкости, легочная дисфункция, осложнения со стороны почек, инсульты и нейрокогнитивные расстройства, угнетение иммунитета, реперфузионный синдром. За последние годы проведен ряд работ направленных на сравнение осложнений после операций коронарного шунтирования выполненных в условиях ИК и на работающем сердце. Эти исследования позволили оценить влияние искусственного кровообращения на число и характер послеоперационных осложнений. $[4,11]$

Искусственное кровообращение является основной причиной интраоперационной микроэмболии и гипоперфузии головного мозга. Одной из работ, направленных на оценку неврологических нарушений, связанных с ИК, стало исследование, проведенное в Германии в Лейпцигском Университете. Сорок пациентов были рандомизированы на 2 группы. Нейрокогнитивный статус оценивался до- и послеоперационным периоде. Также до и после операции определяли в плазме крови уровень высокоспецифичного маркера повреждения головного мозга, протеина S-100, а во время операции выполняли транскраниальную допплерографию средней мозговой артерии. Среднее значение высокоинтенсивных транзиторных сигналов составило 394.5 в группе с ИК по сравнению с 11 в группе пациентов, оперированных на работающем сердце $(p<0.0001)$. Послеоперационный уровень протеина S-100 в группах с ИК и без ИК составил 3.76 мкг/л и 0.13 мкг/л соответственно ( $\mathbf{0}<0.0001)$. Результаты тестов показали снижение нейрокогнитивной функции у $90 \%$ пациентов, оперированных по стандартной методике, и полное отсутствие нейрокогнитивных расстройств в группе малоинвазивной реваскуляризации миокарда. Таким 
Таблица 1. Послеоперационные результаты

(Todd M. Dewey at al. Ann Thorac Surg 2001;72: 788-92)

\begin{tabular}{|l|l|l|l|}
\hline Показатель & Без ИК $(\mathbf{n = 1 0 0 )}$ & С ИК (n=723) & Достоверность \\
\hline ИМ & $1(1 \%)$ & $10(1.4 \%)$ & НД \\
\hline $\begin{array}{l}\text { Инотропная поддержка п/ } \\
\text { опер. }\end{array}$ & $23(23 \%)$ & $454(62.8 \%)$ & 0.001 \\
\hline Трансфузия & $35(35 \%)$ & $483(66.8 \%)$ & 0.001 \\
\hline Эр. масса & $31(31 \%)$ & $437(60.4 \%)$ & 0.001 \\
\hline Тромбомасса & $11(11 \%)$ & $257(35.5 \%)$ & 0.001 \\
\hline Дней п/о & $7.8( \pm 6.4)$ & $7.2( \pm 4.9)$ & НД \\
\hline Летальность & $1(1 \%)$ & $34(4.7 \%)$ & НД \\
\hline
\end{tabular}

*НД - разница не достоверна.

образом, прослеживается четкая связь нейрокогнитивных расстройств с искусственным кровообращением и микроэмболиями, а «оff - puтр» техника позволяет сократить число подобных осложнений $[3,20]$.

В одном из исследований, посвященных влиянию искусственного кровообращения на функцию почек, было установлено следующее: гломерулярная фильтрация, оцениваемая по клиренсу креатинина и соотношению микро альбуминурии и креатинина мочи, значительно хуже в группе стандартного коронарного шунтирования ( $p=0.0004$ и 0.0083 соответственно). К тому же, снижается функция тубулярного аппарата почек, что подтверждается усилением активности N-aцетилглюкозаминидазы. Таким образом, малоинвазивная реваскуляризация миокарда обладает ренопротективным действием по сравнению со стандартной техникой, подразумевающей использование аппарата искусственного кровообращения и кардиоплегическую остановку сердца $[8,19]$

К очевидным преимуществам малоинвазивного коронарного шунтирования относятся значительное снижение кровопотери в предоперационном периоде, а также снижение трансфузии препаратов крови [11].

В последние годы резко возросла доля малоинвазивных вмешательств в структуре операций коронарного шунтирования. В некоторых клиниках до 97\% операций выполняется на работающем сердце. Однако, до недавнего времени, поражение ствола левой коронарной артерии являлось относительным противопоказанием к операциям без искусственного кровообращения, что связано с резким изменением гемодинамики при вывихивании сердца у данной категории больных [20]. Но усовершенствование методов экспозиции миокарда (вакуумные стабилизаторы и вертикализаторы), усовершенствование анестезиологического пособия (использование перидуральной анестезии) привело к появлению ряда статей об успешном выпол- нении операций ОРСАВG у больных со стенозом ствола ЛКА.

В 2001 году Dewey с соавторами опубликовал статью, в которой проводился сравнительный анализ больных с поражением ствола ЛКА, оперированных на работающем сердце и в условиях искусственного кровообращения. С января 1998 по октябрь 1999 года было выполнено 823 операции коронарного шунтирования у больных с гемодинамически значимым стенозом ствола левой коронарной артерии, из них 100 операций выполнено по методике ОРСАВ $[3,16]$. Отбор пациентов не был рандомизированным, в группу оперированных на работающем сердце выбирались больные с высоким риском осложнений искусственного кровообращения. Сюда вошли пациенты пожилого возраста, больные с поражением почек или со сниженной функцией легких, с инсультами в анамнезе или тяжелым поражением брахиоцефальных сосудов, а также пациенты со значительным кальцинозом аорты. В связи с этим между группами существовали определенные различия. Средний возраст пациентов в группе оперированных на работающем сердце составил 67.6 лет против 63.7 лет в группе с использованием ИК (р<0.001), средняя фракция выброса была достоверно ниже в группе стандартной реваскуляризации миокарда (48\% $\pm 12.6 \%$ против 52\% $12.5 \%$, коэффициент достоверности 0.013). Дооперационная дисфункция почек значительно чаще встречалась в группе ОРСАВ ( $p=0.003)$. Во время операции большинству пациентов в обеих группах было выполнено 3 и более шунтов. Индексы реваскуляризации в группах ОРСАВ и с использованием искусственного кровообращения составили 3.4 и 2.87 соответственно.

Результаты, полученные авторами, в ходе исследования приведены в таблице 1.

Не было выявлено достоверной разницы в послеоперационных осложнениях, в обеих группах было зафиксировано малое количество инсультов, число 
легочных и инфекционных осложнений также было сопоставимо.

В результате, отсутствие достоверной разницы в показателях летальности и послеоперационных осложнений между двумя группами, свидетельствует о безопасности операций на работающем сердце у больных с поражением ствола левой коронарной артерии. Кроме того, хотя разница и не достоверна, видна тенденция к снижению летальности в группе OPCABG. A накопление опыта выполнения подобных операций может привести к еще большему снижению этого показателя. $[4,13,21]$

К преимуществам операций на работающем сердце, выявленным в данном исследовании, относятся: меньшее число случаев использования инотропных средств в послеоперационном периоде, снижение числа трансфузий препаратов крови (эритроцитарной массы, тромбомассы) [6,22].

В том же 2001 году вышла статья группы авторов под руководством Mark Yeatman из Бристольского Института Сердца, в которой были представлены результаты оперативного лечения больных с поражением ствола ЛКА на работающем сердце и с использованием аппарата искусственного кровообращения. В исследование вошли 387 пациентов, из них в группу ОРСАВ 75 человек и 312 группу стандартного коронарного шунтирования. Пре- и интраоперационные данные у этих больных не отличались, за исключением индекса реваскуляризации, который составил $3.1 \pm 0.73$ в группе

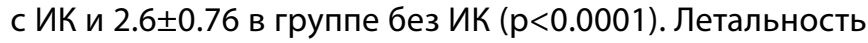
не имела статистически значимых отличий и составила 1.3\% в группе ОРСАВG $(n=75)$ и 2.6\% в группе ИК $(n=312)$. Среди пациентов, оперированных без ИК, отмечено меньшее использование инотропных препаратов (12\% против $38.1 \% \mathrm{P}=0.0001)$, временной стимуляции серд- ца (2.7\% против $10.1 \% \mathrm{P}=0.02)$, а также гемотрансфузий (6.7\% против 31.4\% P <0.0001). В группе OPCABG выявлено меньше случаев послеоперационной инфекции грудины (0\% против 6.7\% P=0.02). Через 24 месяца после проведения операции средняя выживаемость со-

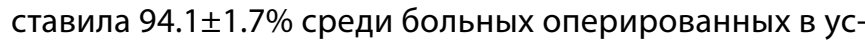
ловиях ИК и $97.7 \pm 2.3 \%$ в группе без ИК. По результатам исследования, авторами сделан вывод о безопасности и эффективности операций на работающем сердце у больных с поражением ствола левой коронарной артерии $[5,7,12,18]$

В ряде исследований других авторов, также сравнивающих результаты операций OPCABG и операций с использованием ИК у больных с гемодинамически значимым сужением ствола левой коронарной артерии (Cartier et al. 1999; Mujanovic et al. 2004), выявлено отсутствие достоверной разницы в показателях летальности и послеоперационных осложнений, а также подтверждены вышеперечисленные преимущества операций ОРСАВ.

Больные с поражением ствола левой коронарной артерии, по-прежнему, остаются группой, требующей повышенного внимания при лечении. Преимущества коронарного шунтирования у подобных больных очевидны, однако, накопление опыта выполнения эндоваскулярной ангиопластики, а также появление новых стентов с покрытиями, может, со временем, поставить под сомнение подобное утверждение. Снижение травматичности операций коронарного шунтирования за счет отказа от искусственного кровообращения является наиболее перспективной и привлекательной методикой для пациентов и хирургов. Необходимо дальнейшее изучение безопасности и эффективности операций на работающем сердце, однако уже сейчас ясно, что становится все меньше противопоказаний для данного вида вмешательства.

\section{ЛИТЕРАТУРА}

1. Белов Ю.В., Лысенко А.В., Комаров Р.Н., Стоногин А.В. Результаты коронарного шунтирования без искусственного кровообращения у пациентов со стенозом ствола левой коронарной артерии и его эквивалентом. Кардиология и сердечно-сосудистая хирургия. - 2016; -vol 5-P. 18-22.

2. Бокерия Л.А., Алекян Б.Г., Бузиашвили Ю.И., Голухова Е.З., Никитина Т.Г., Мерзляков В.Ю., Алшибая М.М., Стаферов А.В., Закарян Н.В., Сравнение результатов чрескожного коронарного вмешательства и аортокоронарного шунтирования при поражении незащищенного ствола левой коронарной артерии. Грудная и сердечно-сосудистая хирургия. - 2013. -vol 6 -P. 33-40.

3. Бабакулова Н.М., Желихажева М.В., Ибрагимов Р.Г., Ключников И.В., Мерзляков В.Ю. Аортокоронарное шунтирование на работающем сердце при критическом поражении ствола левой коронарной артерии: безопасность, эффективность, результат. Анналы хирургии. - 2014.—vol. 3 -P. 26-31.

4. Казанчан П.О., Попов В.А., Сотников П.Г., Козорин М.Г., Циклин И.Л. Миниинвазивная васкуляризация миокарда у больных со стенозом ствола левой коронарной артерии. Кардиология и сердечно-сосудистая хирургия. - 2010 -vol 3-P. 7-12.

5. August P, Brooks MM, Hardison RM, Kelsey SF, MacGregor JM, Orchard TJ, Chaitman BR, Genuth SM, Goldberg SH, Hlatky MA, Jones TL, Molitch ME, Nesto RW, Sako EY, Sobel BE. A randomized trial of therapies for type 2 diabetes and coronary artery disease. N Engl J Med. — 2009. — vol.360 — P. 2503-2515.

6. Ahn JM, Roh JH, Kim YH, et al. Randomized trial of stents versus bypass surgery for left main coronary artery disease: 5-year outcomes of the PRECOMBAT study. J Am Coll Cardiol -2015. - vol .65-P. 2198-206. 
7. El-Menyar AA, Al Suwaidi J, Holmes DR Jr. Left main coronary artery stenosis: state-of-the-art. Curr Probl Cardiol -2007. —vol 32-P. 103-93.

8. Gasior M, Zembala M0, Tajstra M, Filipiak K, Gierlotka M, Hrapkowicz T, Hawranek M, Polonski L, Zembala M. Hybrid revascularization for multivessel coronary artery disease. JACC Cardiovasc Interv. - 2014. -vol 7. -P 1277-1283.

9. Mäkikallio T, Holm NR, Lindsay M, et al. Percutaneous coronary angioplasty versus coronary artery bypass grafting in treatment of unprotected left main stenosis (NOBLE): a prospective, randomised, open-label, non-inferiority trial. Lancet -2016 -vol. 388:2743.

10. Mohr FW, Morice MC, Kappetein AP, et al. Coronary artery bypass graft surgery versus percutaneous coronary intervention in patients with three-vessel disease and left main coronary disease: 5-year follow-up of the randomised, clinical SYNTAX trial. Lancet -2013. -vol 381 -P. 629-638.

11. Morice MC, Serruys PW, Kappetein AP, et al. Five-year outcomes in patients with left main disease treated with either percutaneous coronary intervention or coronary artery bypass grafting in the synergy between percutaneous coronary intervention with taxus and cardiac surgery trial. Circulation -2014. - vol. 129 -P. 2388.

12. Morice MC, Serruys PW, Kappetein AP, et al. Five-year outcomes in patients with left main disease treated with either percutaneous coronary intervention or coronary artery bypass grafting in the synergy between percutaneous coronary intervention with taxus and cardiac surgery trial. Circulation -2014. - vol 129. - P. 2388-2394.

13. Park SJ, Kim YH, Park DW, et al. Randomized trial of stents versus bypass surgery for left main coronary artery disease. N Engl J Med -2011.—vol. 364 P. $1718-27$.

14. Park SJ, Kim YH, Park DW, Lee SW, Kim WJ, Suh J, Yun SC, Lee CW, Hong MK, Lee JH, Park SW. Impact of intravascular ultrasound guidance on long-term mortality in stenting for unprotected left main coronary artery stenosis. Circ Cardiovasc Interv. — 2009 — vol. 2 -P167-177.

15. Ragosta M, Dee S, Sarembock IJ, et al. Prevalence of unfavorable angiographic characteristics for percutaneous intervention in patients with unprotected left main coronary artery disease. Catheter Cardiovasc Interv -2006 - vol 68 - P. 357-366.

16. Serruys PW, Morice MC, Kappetein AP, Colombo A, Holmes DR, Mack MJ, Stahle E, Feldman TE, van den Brand M, Bass EJ, Van Dyck N, Leadley K, Dawkins KD, Mohr FW. Percutaneous coronary intervention versus coronary-artery bypass grafting for severe coronary artery disease. N Engl J Med. — 2009. — vol. 360 — P. 961-972.

17. Serruys PW, Morice MC, Kappetein AP, et al. Percutaneous coronary intervention versus coronary-artery bypass grafting for severe coronary artery disease. $\mathrm{N}$ Engl J Med -2009; -vol. 360 - P. 961-967.

18. Stone GW, Kappetein AP, Sabik JF, et al. Five-Year Outcomes after PCI or CABG for Left Main Coronary Disease. N Engl J Med — 2019 -vol. 381 -P. 1820.

19. Taggart DP, Altman DG, Gray AM, Lees B, Gerry S, Benedetto U, Flather M. Randomized trial of bilateral versus single internal-thoracic-artery grafts. N Engl J Med.-2016. - vol. 375 -P. $2540-2549$

20. Taggart DP, Kaul S, Boden WE, et al. Revascularization for unprotected left main stem coronary artery stenosis stenting or surgery. J Am Coll Cardiol -2008. vol.51 - P. 885-891.

21. Windecker S, Kolh P, Alfonso F, et al. 2014 ESC/EACTS Guidelines on myocardial revascularization: The Task Force on Myocardial Revascularization of the European Society of Cardiology (ESC) and the European Association for Cardio-Thoracic Surgery (EACTS)Developed with the special contribution of the European Association of Percutaneous Cardiovascular Interventions (EAPCI). Eur Heart J — 2014 — vol. 35. —P. 2541-619.

22. Yusuf $S$, Zucker D, Peduzzi P, et al. Effect of coronary artery bypass graft surgery on survival: overview of 10 -year results from randomised trials by the Coronary Artery Bypass Graft Surgery Trialists Collaboration. Lancet -1994. — vol. 344 — P. 563-570

( Ж Жалилов Адхам Кахрамонович ( Jalilov_adham@mail.ru). Журнал «Современная наука: актуальные проблемы теории и практики» 